\title{
MIRIDAE (HETEROPTERA) FITÓFAGOS E PREDADORES DE MINAS GERAIS, BRASIL, COM ÊNFASE EM ESPÉCIES COM POTENCIAL ECONÔMICO
}

\author{
Paulo S. F. Ferreira ${ }^{1}$ \\ Elidiomar R. da Silva ${ }^{2}$ \\ Luci B. N. Coelho ${ }^{3}$
}

\begin{abstract}
PHYTOPHAGOUS AND PREDACEOUS MIRIDAE (HETEROPTERA) OF MINAS GERAIS, BRAZIL, WITH EMPHASIS ON POTENTIAL PEST SPECIES. A total of 296 species of Miridae belonging to 17 tribes were recorded from State of Minas Gerais, Brazil. New host plant records include 141 species of 43 orders and 69 families. Of this total, $98.5 \%$ of plants have some agricultural, pharmacological or ornamental importance; 29 orders and families of host plants have two or more mirid species.
\end{abstract}

KEYWORDS. Neotropical, Miridae, host plants, Minas Gerais, Brazil.

\section{INTRODUÇÃO}

Miridae é um táxon que corresponde a maior família de Heteroptera, com aproximadamente 10000 espécies dentro de 1383 gêneros ao redor do mundo (SCHUH, 1995). A Região Neotropical, representada por aproximadamente 3000 espécies, possui o dobro do número registrado para a Região Neártica (HenRY \& Froeschner, 1988; Schuн, 1995). No Brasil são conhecidas cerca de 1000 espécies, número baixo considerando-se o tamanho territorial e a diversidade florística que o colocam em primeiro lugar na biodiversidade mundial. Para muitos estados brasileiros, principalmente aqueles da região Nordeste, a diversidade de mirídeos é praticamente desconhecida. Minas Gerais é o estado que apresenta a maior riqueza documentada, com 155 espécies.

Os mirídeos têm sido alvo de estudos pelos danos que causam às plantas cultivadas (WhEELER, 2000a), pela presença de espécies predadoras com potencial para agentes de controle biológico (WHEELER, 2000b), e pelo fato biológico de que grande número de espécies fitosuccívoras, conhecidas como "facultativas" ou omnívoras, apresentar, ocasionalmente, hábitos predatórios (HeNRY, 2000). A demanda por identificação de mirídeos para pesquisas relacionadas às plantas cultivadas tem se intensificado. Muitas espécies têm sido identificadas como novas, como novos registros para plantas hospedeiras e como ocorrência inédita em regiões brasileiras. Isto sugere mudanças comportamentais de espécies fitosuccívoras. Trata-se de uma dinâmica crescente, possivelmente acompanhando as mudanças tecnológicas nos tratamentos agrícolas.

Objetiva-se ampliar o conhecimento da riqueza de mirídeos do Estado de Minas Gerais, ampliar o registro de novas plantas hospedeiras e levantar hipótese sobre possíveis plantas que poderão ser hospedeiras potenciais.

1. Universidade Federal de Viçosa, Departamento de Biologia Animal, 36571-000, Viçosa, MG, Brasil. (pfiuza@mail.ufv.br)

2. Departamento de Ciências Naturais, Escola de Ciências Biológicas, UNI-RIO, rua Frei Caneca, 94, $5^{\circ}$ andar, 20211-040, Rio de Janeiro, RJ, Brasil. (elidiomar@bol.com.br)

3. Departamento de Zoologia, Instituto de Biologia, UFRJ. Caixa Postal 68044, 21944-970, Rio de Janeiro, RJ, Brasil. (lucibncoelho@bol.com.br) 


\section{MATERIAL E MÉTODOS}

Foram coletados ao redor de 500 espécimes de mirídeos durante os períodos de novembro a fevereiro de 1998 e 1999, nos municípios de Campos Altos, Dores do Indaiá, Florestal e São Gotardo. Para a coleta utilizou-se rede de varredura e armadilha luminosa "black-light" (FERREIRA \& MARTINS, 1982). As plantas foram amostradas e identificadas pelo Departamento de Biologia Vegetal da Universidade Federal de Viçosa (UFV). O material entomológico encontra-se depositado no Museu de Entomologia do Departamento de Biologia Animal (UFV).

Foram utilizados dados da literatura especializada e de aproximadamente 2400 exemplares adultos depositados em coleções entomológicas do Museu Nacional, Universidade Federal do Rio de Janeiro (MNRJ) e do Museu Regional de Entomologia da Universidade Federal de Viçosa (UFVB). As espécies consideradas para o Estado de Minas Gerais foram encontradas nos seguintes topônimos mineiros: Águas Claras, Águas Vermelhas, Arapongas, Araxá, Belo Horizonte, Caetés, Campos Altos, Capinópolis, Carmo do Rio Claro, Cataguases, Caxambu, Coimbra, Coronel Fabriciano, Delfim Moreira, Dores do Indaiá, Ervália, Florestal, Itabirito, Itamonte, Juiz de Fora, Lagoa Santa, Ouro Preto, Paracatu, Paula Cândido, Pedra Azul, Pirapora, Piuí-Caraças, Ponte Nova, Raul Soares, Rio Teles Pires, Rio Doce, São Gotardo, Sete Lagoas, Sinop,Varginha e Viçosa.

As citações dos autores para os nomes das plantas foram baseadas na obra de BRUMmitT \& Powell (1996).

\section{RESULTADOS E DISCUSSÃO}

Foram constatadas 296 espécies de Miridae para o Estado de Minas Gerais, distribuídas em 17 tribos. A expressiva quantidade de novos registros evidencia a necessidade crescente de se incentivar a realização de levantamentos entomofaunísticos em diversas regiões do Brasil. E isto se aplica em especial a grupos com potencial econômico, como as pragas de culturas, que naturalmente são mais estudados. Acrescentam-se 141 espécies para as 155 conhecidas de Minas Gerais, quase que duplicando o número previamente registrado. As tribos com suas espécies e plantas hospedeiras conhecidas e em potencial, além de novos registros para o estado são pela primeira vez apresentados (tab. I). As tribos mais representativas (tab. I) em número de espécies foram, em ordem decrescente: Mirini (91), Orthotylini (52), Eccritotarsini (44), Resthenini (21) e Phylini (19). Todas essas tribos contêm, na sua grande maioria, espécies conhecidamente de hábito fitosuccívoro.

A maioria das espécies depositadas em museus não apresenta informações sobre suas plantas-hospedeiras. No entanto, referências às espécies em escala mundial têm demonstrado uma tendência dos mirídeos de serem "especialistas" para gêneros e famílias de plantas (SСHuH, 1995). Por esta razão, considerou-se a hipótese de que os gêneros fitosuccívoros se restringem a certas espécies vegetais, sugerindo que possam representar grupos taxonômicos filogeneticamente associados. Assim, gêneros de mirídeos brasileiros ocorrentes em Minas Gerais e sem registro de plantas hospedeiras, mas que são encontrados em outras localidades com plantas hospedeiras de distribuição no Brasil, estes vegetais foram considerados "plantas hospedeiras em potencial" (tab. I).

No total, 69 famílias de plantas, representantes de 43 ordens, foram registradas como hospedeiras ou potenciais hospedeiras de espécies de Miridae, das quais 68 apresentam alguma importância econômica (Gemtchúsnicov, 1976; Schultz, 1990), seja como cultivar agrícola, ornamental ou de uso medicinal (tab. II). Destas, 29 famílias apresentam duas ou mais espécies susceptíveis à colonização por parte dos Miridae (fig. 1), com destaque para as famílias Fabaceae (18), Gramineae (17), Asteraceae (16) e Orchidaceae (9). Com relação às ordens (fig. 2), 29 delas têm mais de uma espécie hospedeira potencial, destacando-se Fabales (18), Poales (17), Asterales (16), Orchidales 
(9), Gentianales (9) e Caryophyllales (8). No total, 31 famílias são colonizadas por dois ou mais gêneros de Miridae (fig. 3), sobressaindo-se Fabaceae (23 gêneros), Asteraceae (13), Solanaceae (12), Malvaceae (11), Gramineae (10), Convolvulaceae (10) e Euphorbiaceae (9).

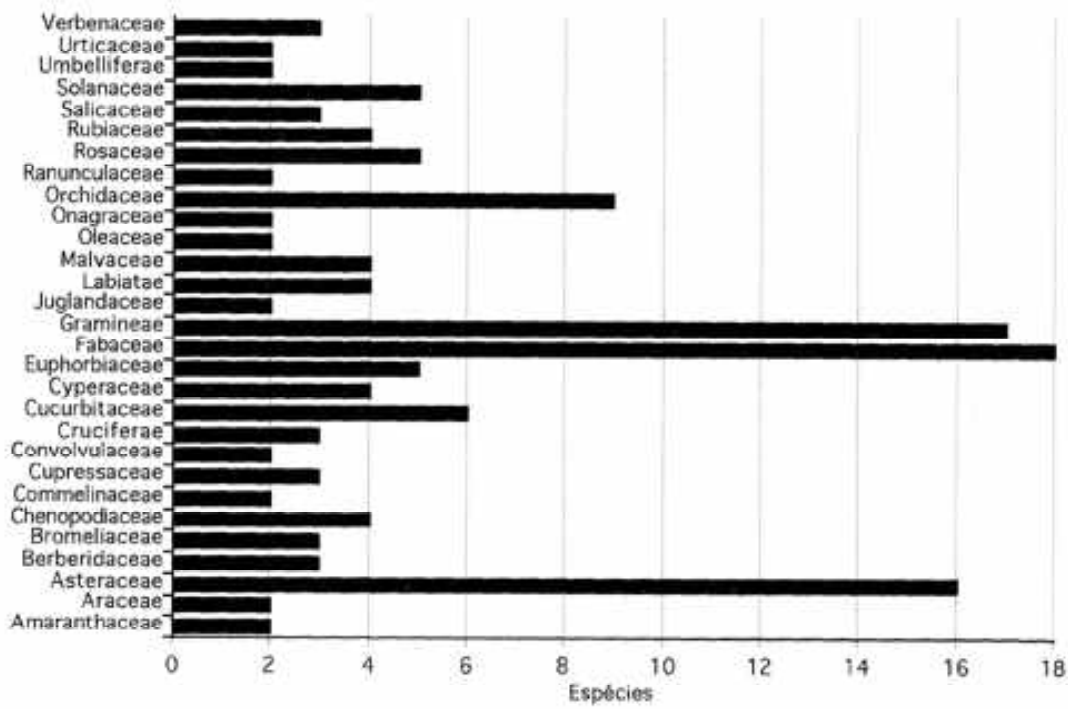

Fig. 1. Famílias de plantas-hospedeiras de Miridae, Minas Gerais, e número de espécies de mirídeos. Somente foram incluídas famílias com duas ou mais espécies.

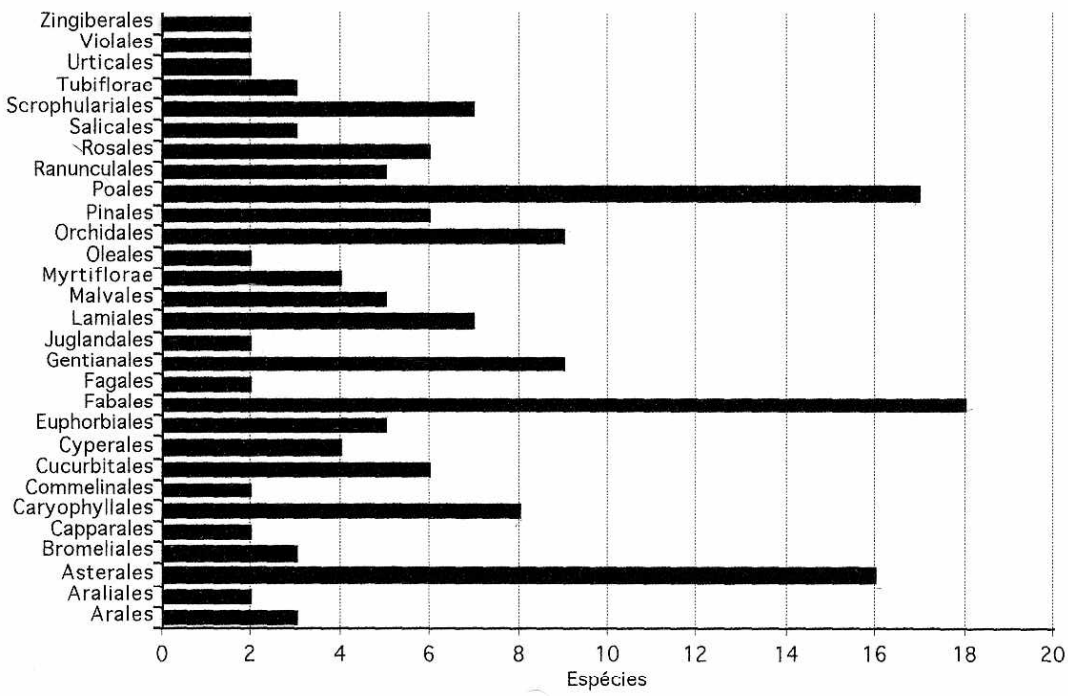

Fig. 2. Ordens de plantas-hospedeiras de Miridae, Minas Gerais, e número de espécies de mirídeos. Somente foram incluídas ordens com duas ou mais espécies. 
Tabela I. Relação dos Miridae coletados em 1998 e 1999 e referidos em bibliografia para o Estado de Minas Gerais, Brasil. (* novo registro; plantas sublinhadas $=$ hospedeiros potenciais).

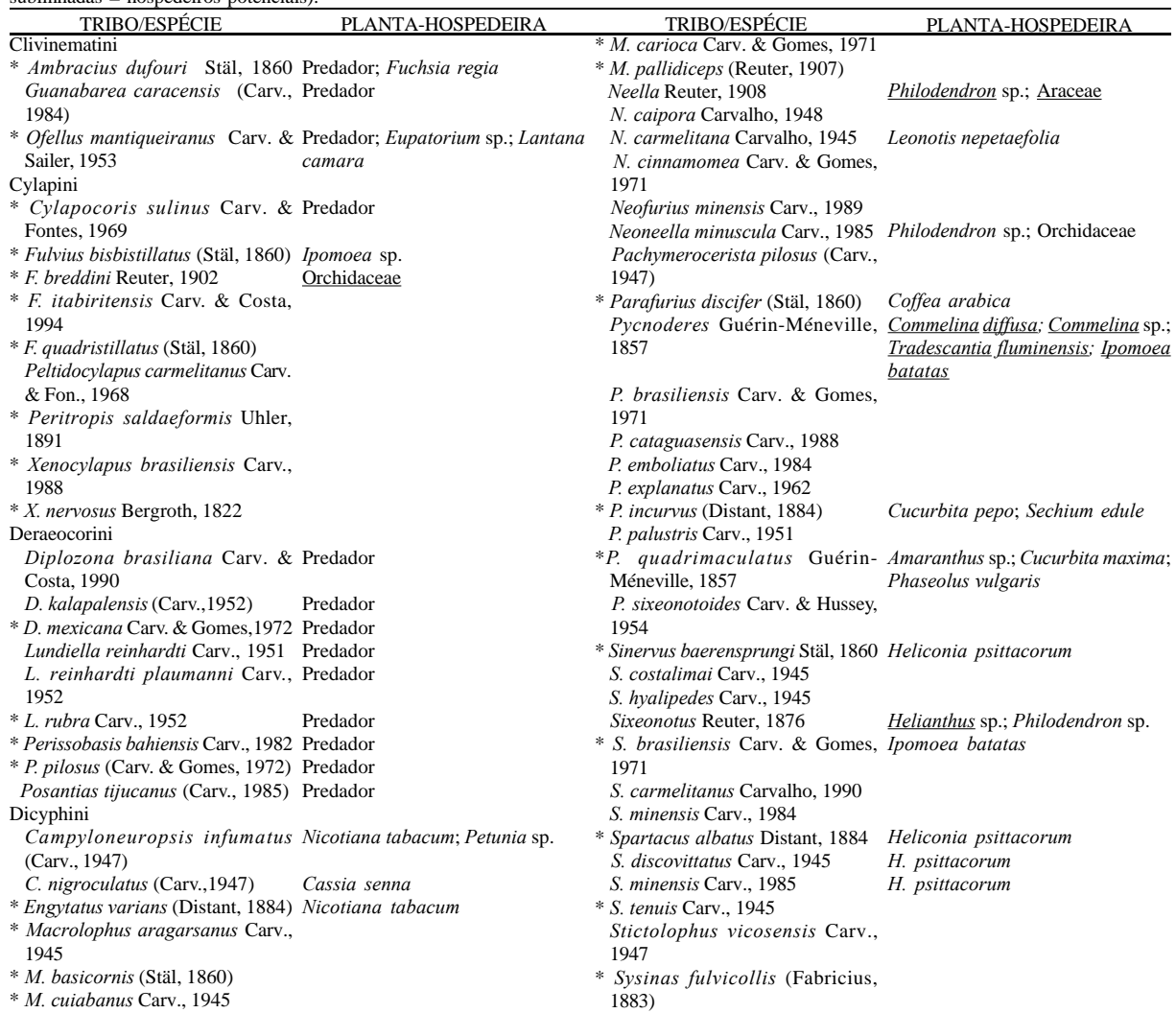

* M cuiabanus Carv., 1945

* M. praeclarus (Distant, 1893) Nicotiana tabacum

* Tupiocoris cucurbitaceus Lycopersicon esculentum

Tenthecoris Scott, 1886 (Spinola, 1852)

* T. notatus (Uhler, 1893)

Eccritotarsini

* Aspidobothrus dimidiatus (Stäl, Thevetia sp. 1860)

* T. hsiaoi Carv., 1948

* T. nanus Carv., 1948

Cattleya sp.; Deudrobium sp.; Epidendrum sp.; Gomeza sp.; Lycaste sp.; Oncidium sp.; Primavera $\mathrm{sp}$.

* Bothrophorella nigra (Stäl, 1860) Ipomoea batatas Cyrtocapsus Reuter, $1876 \quad$ Ananas comosus; Ipomoea batatas; Halticini

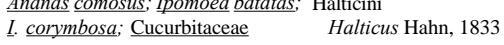

* C. femoralis Reuter, 1892 Eccritotarsus Stäl, 1860 E. brasiliensis Carv. \& Gomes, 1971

* E. compactus Carv., 1953

*E. corcovadensis Carv. \& Schaffner, 1986

* E. cruxnigra Stäl, 1860 E. hyalinus Stäl, 1860

* E. nigrocruciatus Stäl, 1860

Eurychilella Reuter, 1908

* E. discoidalis (Reuter, 1912) E. paracatua Carv., 1984 Eurychiloides bilobosus Carv. \& Gomes, 1971 Monalocoris Dahlbom, 1851 Colocasia esculenta; Erythrina sp.

Caladium sp.

Aclandiae sp.; Cattleya labiata; Laelia perrinii

Ipomoea batatas; Cucumis melo reticulata; Cucurbita moschata; Phaseolus vulgaris; Vicia sp.; Anemone sp.; Clematis sp.; Cynodom dactylon Brassica napus; Brassica sp.; Petroselinum sativum

Philodendron sp.; Billbergia sp. Caladium $\mathrm{sp}$.

* H. bracteatus (Say, 1832

Herdoniini

Adxenetus falloui (Poppius, 1921)

A. minensis Carv. \& Ferreira, 1974

* A. petiolatus (Stäl, 1860)

Allommatela rugosa Carv. \&

Ferreira, 1986

Haarupia minuscula Carv. \& Ferreira, 1974

* Paraxenetus annulicornis Reuter, 1907

Dryopteris sp.; $\underline{\text { Pteridium }}$ aquilinum 
Tab. I. Continuação

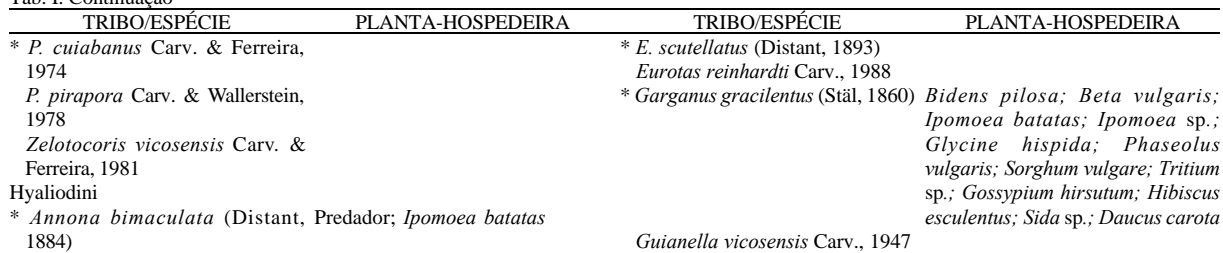

1884)

A. brasiliensis Carv. \& Schaffner, Predador 1977

Henicocnemis carmelitanus

Carv., 1985

* A. fuscata Carv. \& Schaffner, 1977 Predador

* Brasiliocarnus sulinus (Carv. \& Predador

* H. patellata Stäl, 1860

Horcias Distant, 1884

Gomes, 1971) H. carmelitanus Carv., 1987

Carijoanus ruberfasciatus (Carv., Predador; Cecropia sp.; $\quad$ H. guapeanus Carv., 1976

1945)

H. guapeanus Carv., 1976
H. lineifer Carv., 1976

Hyaliodes Reuter, 1876

* H. glabratus Distant, 1888

H. intercallosus Carv., 1984

Monimiaceae

* H. lineifer Carv., 1976
H. minensis Carv., 1976

$\begin{array}{ll}\text { Predador; Corylus sp.; Quercus sp. } & \text { H. minensis Carv., } 1976 \\ \text { Predador; } \text { Manihot utilissima } & \text { H. nobilellus (Berg, 1883) }\end{array}$

H. minensis Carv., 1984

Predador

Predador; Manihot utilissima

* H. vitreus (Distant, 1884)

Predador
Predador

H. wygodzinskyi Carv., 1945

$\begin{array}{ll}* \text { Hyaliodocoris clarus (Stäl, 1860) Predador; Cayaponia diversifolia } \\ \text { * H. insignis (Stäl, 1860) } & \text { Predador; Phaseolus vulgaris }\end{array}$

Mirini

* Bahiamiris rubrornatus Carv.,

1975

Chrysodasia caracensis Carv., 1986

* C. manifesta (Distant, 1893)

Creontiades Distant, 1883

Heliotropium curassavicum;

Indigofera tinctoria; Quercus sp.; * Lampethusa vingtuni Carv., 1990

Piper sp. Liliancoris nigrus Carv., 1989

C. purgatus (Stäl, 1860)

* C. rubrinervis (Stäl, 1860)

Ipomoea sp.; Zea mays; Daucus carota

Minasmiris jugatus Carv., 1980

Ipomea sp.; Canavalia $\quad$ Monalocorisca Distant, 1884

Borreria sp.

Ipomoea sp.; Gossypium hirsutum

Phaseolus vulgaris; Phaseolus sp.; M. nordestina Carv., 1989

Vigna sinensis; Gossypium Neostenotus bracatinganus Carv., hirsutum 1985

Dagbertus Distant, 1904

Tecoma stans; $\underline{\text { Persea }}$ gratissima

Eugenia $\mathrm{sp}$

1985

H. notatus Distant, 1884
H. ouropretanus Carvalho, 1989

* H. vittatus Carv., 1976

* Horciasinus signoreti (Stäl, 1859) Beta vulgaris; Phaseolus vulgaris; Gossypium hirsutum; Daucus carota

Amaranthus spinosus; Bidens pilosa; Gossypium hirsutum; Hibiscus esculentus; Malvastrum coromandelianum; Sida cordifolia; Triumfeta semitriloba

* D. bonariensis (Stäl, 1859)

elongatus Carv., 1990

* D. carmelitanus Carv. \& Fontes, 1983

* D. diamantinus Carv., 1984

D. emboabanus Carv., 1985

D. guaraniensis Carv. \& Fontes, 1983

* D. insignis Carv., 1977

D. minensis Carv. \& Fontes, 1983

* D. obscurus Carv. \& Fontes, 1983

* D. phaleratus (Berg, 1892)

* Derophthalma coriaria Knight \&

Carv., 1943

* D. fluminensis Carv., 1944

* D. guaraniana Carv. \& Gomes, 1980

D. minuscula Carv., 1944

* D. neotropica Carv. \& Gomes, 1980

* D. reuteri Berg, 1883

* Eglerocoris egleri Carv. \& Fontes, 1972

Esavicoris esavianus (Carv., 1953)

Eubatas pirapora Carv. \&

Wallerstein, 1978

* Euchilocoris rufinasus (Stäl, 1860)

N. fuscipennis (Reuter, 1909)

N. nigroviridis Carv. \& Ferreira, 1986

Notholopus carmelitanus Carv. \&

Fer., 1971

N. sulcaticornis (Stäl, 1860)

Phytocoris Fallen, 1814

Predador; Ambrosia sp.; Artemisia sp.; Baccharis sp.; Echinops sp.; Haplopappus sp.; Santolina sp.; Tanacetum vulgare; Berberis cretica; Berberis fremontii; Berberis trifoliata; Corylus sp.; Tillandsia usneoides; Buxus sempervirens; Symphoricarpos sp.; Atriplex sp.; Chenopodium sp.; Suaeda sp.; Cistus villosus; Hypericum sp.; Callitris sp.; Cupressus sp.; Juniperus sp.; Pinus sp.; Podocarpus sp.; Ephedra sp.; Acacia sp.; Prosopis juliflora; Quercus suber; Juglans regia; Rosmarinus officinalis; Salvia sp.; Thymus vulgaris; Phoradendron sp.; Malvastrum sp.; Olea europaea; Platanus sp.;_Limonium sp.; Malus sylvestris; Pyrus sp.; Rubus fructicosus; $\underline{\text { Coffea }}$ sp.; Salix sp.; Verbascum sp.; Tamarix sp.; Lantana sp.; Verbena sp.

Continua

Iheringia, Sér. Zool., Porto Alegre, (91): 159-169, 27 de novembro de 2001 
Tab. I. Continuação

\begin{tabular}{|c|c|}
\hline \multirow{2}{*}{\multicolumn{2}{|c|}{ TRIBO/ESPECIE }} \\
\hline & \\
\hline * P. aspersus Carv. \& Gom & \\
\hline P. bellissimus Carv. \& & \\
\hline 1986 & \\
\hline * P. bergrothi Reuter, 1892 & \\
\hline P. clarensis Carv. \& Costa & \\
\hline * P. cylapinus Carv. \& Gom & \\
\hline * P. effictus Stäl, 1860 & \\
\hline P. fiuzai Carv. \& Costa, 19 & \\
\hline P. minensis Carv., 1986 & \\
\hline * P. subvittatus (Stäl, 1860) & \\
\hline P. translucidus Carv. \& F & \\
\hline 1986 & \\
\hline * Phytocorisca minima & \\
\hline Fontes, 1972 & \\
\hline * P. ocellata Carv. \& Fonte & \\
\hline P. vissosensis Carv. \& & \\
\hline 1986 & \\
\hline * Piasus cribricollis (Stäl, & \\
\hline * Poeas serrana Carv., 19? & \\
\hline Polymerus Hahn, 1831 & \\
\hline *P. aristeae Ferreira, 1980 & \\
\hline * P. caligatus $($ Stäl, 1860) & \\
\hline *P. minutus Ferreira, 1979 & \\
\hline * P. testaceipes (Stäl, 1860 & \\
\hline
\end{tabular}

Proba Distant, 1884

P. missionera Carv. \& Carpintero, 1986

* Proba vittiscutis (Stäl, 1860)

* Taedia bimaculata (Fabricius, 1803)

T. clarensis Carv. \& Costa, 1993

* T. compactoides Carv., 1975

* T. distantina Carv., 1954

* T. leprosa (Walker, 1873)

* T. nobilitata (Stäl, 1860)

T. pirapora Carv. \& Costa, 1993

T. scutellata Carv. \& Wallerstein, 1978

* T. semilota (Stäl, 1860)

* T. signata Carv. \& Gomes, 1971

* T. similaris Carv. \& Gomes, 1971

T. stigmosa (Berg, 1878)

Taylorilygus Leston, 1952

* T. apicalis (Blanchard, 1852)

Tropidosteptes Uhler, 1878

* T. cribratus (Stäl, 1860)

* T. hirsutus (Distant, 1884)

* T. lineatus Carv., 1986

Vissosamiris brasiliensis (Carv. 1955)

Monaloniini

* Monalonion bahiense Lima, 1938 Theobroma cacao

Orthotylini

Adfalconia cunealis Carv. \& Acalypha brasiliensis

Rosas, 1962

Adhyalochloria itatiaiensis

(Carv., 1986)

Predador

Predador

Predador

Predador

Predador

Predador

Persea gratissima Daucus carota hirsutum

Gossypium hirsutum sp.

\begin{tabular}{lcc}
\multicolumn{1}{c}{ PLANTA-HOSPEDEIRA } & TRIBO/ESPÉCIE & PLANTA-HOSPEDEIRA \\
\hline Predador & $*$ Adparaproba binotata (Carv. \& & \\
Predador & Fer., 1987) \\
Predador & $*$ A. dispersa Carv., 1987 &
\end{tabular}

Phaseolus vulgaris, Mimosa sp.

A. gabrieli Carv., 1987

A. piranga Carv., 1987

Brasiliomiris ernestoi Carv., Cecropia sp.

1947

Ceratocapsus emboabanus Carv.

\& Fontes, 1983

C. esavianus Carv. \& Ferreira,

1986

C. liliae Carv. \& Ferreira, 1986

C. minensis Carv. \& Fontes, 1983

* C. ouropretanus Carv. \& Costa, 1990

C. riodocensis Carv. \& Fontes, 1983

C. vissosensis Carv. \& Ferreira,

1986

Artemisia sp.; Erigeron sp.; Solidago sp.; Arenaria sp.; Medicago sativa; Phlox sp.

Fontes, 1983

* Cyrtotylus rubricatus Bergroth, 1922

Esavia vissosensis Carv. \& Ferreira, 1986

Eucerella robusta (Carv. \&

Amaranthus sp.; Amaranthus spinosus; Helianthus sp.; Parthenium sp.; Cleome sp. Bidens pilosa; Dahlia sp., Gossypium sp.; Lycopersicum esculentum; Nicotiana tabacum;

Baccharis sp.; Gossypium

Ferreira, 1986)

Falconia Distant, 1884

* F. costae (Stäl, 1860)

F. guaraniana Carv., 1987

F. varicolor Carv., 1945

Falconisca vissosensis Carv. \& Ferreira, 1986

Hadronemella piraporensis Carv.

\& Costa, 1992

H. vermelhensis Carv. \& Costa, 1992

Hyalochloria Reuter, 1907

Gossypium sp.; Sida sp.; Ipomoea

Hyalochloria brasiliana Henry, 1968

Jobertus esavianus Carv., 1944

J. gabrieli Carv., 1987

Laemocoridea brasiliensis Carv., 1988

* L. dispersa (Carv., 1944)

L. punctata (Carv. \& Rosas,1965)

Orthotylus Fieber, 1858

Euphorbiaceae; Inga sp. sp.; Lantana sp.

Cucurbita moschata; Phaseolus vulgaris; Clerodendrum sp.

Combretum sp.; Caucanthus auriculatus; Boerhavia sp.; Rumex sp.; Salix sp.; Balanites aegyptica Beta vulgaris; Daucus carota

Quercus sp.; Osmanthus sp.; Malus

O. angeloi Carv., 1986
* O. aureopubescens (Carv. \&
Schaffner, 1974)
* O. bahianus Carv., 1976
O. costai Kerzhner \& Schuh,
1995
O. cyanescens Carv. \& Ferreira,
1986
O. esavianus Carv. \& Fer., 1986
* O. josei Kerzhner \& Schuh, 1995

Antennaria sp.; Achillea sp.; Corylus sp.; Symphoricarpos sp.; Atriplex sp.; Chenopodium sp.; Salicornia sp.; $\underline{\text { Suaeda sp.; Calitris sp.; Cupressus }}$ sp.; Juniperus sp.; Pinus sp.; Taxodium distichum; Acacia sp.; Indigofera sp.; Spartium sp.; Tamarindus indica; Quercus sp.; Carya illinoensis; Juglans sp.; Populus nigra; Populus tremula; Salix sp.; Tamarix sp.; Urtica sp.

Iheringia, Sér. Zool., Porto Alegre, (91): 159-169, 27 de novembro de 2001 
Tab. I. Continuação

\begin{tabular}{l}
\hline \multicolumn{1}{c}{ TRIBO/ESPÉCIE } \\
\hline O. membranosus (Carv. \& Costa, \\
1992) \\
O. minensis (Carv., 1985) \\
O. nigroluteus Carv. \& Ferreira, \\
1986 \\
O. riodocensis Carv., 1986 \\
O. vanetti Carv. \& Ferreira, 1987 \\
O. vermelhensis (Carv. \& Costa, \\
1992) \\
Paraproba Distant, 1884
\end{tabular}

P. binotata Carv. \& Ferreira, 1986 P. brasiliana Carv. \& Ferreira, 1987

Saileria Hsiao, 1945

S. almeidai (Carv., 1946)

S. carmelitana Carvalho, 1991

* S. serrana Carv., 1985

Sericophanes ornatus (Berg, Medicago sativa 1878)

* S. scotti (Berg, 1883)

Solanocoris semiruber Carv., Solanum verbascifolium 1945

Vanettia rubra Carv. \& Ferreira,

1986

Phylini

Botocudomiris clypeatus Carv., 1979

* Crassicornus pulchrus Carv., 1945

Hyalopsallus diaphanus (Reuter,

1907)

* Lepidopsallus riodocensis Carv., 1980

* Moissonia cuneata (Stäl, 1860)

* Nigrimiris pallidipes Carv. \&

Schaffner, 1974

Platyscytus Reuter, 1907

P. decempunctatus (Carv., 1945)

* Platyscytus montei (Carv., 1945)

P. paulistanus (Carv., 1945)

P. rufoscutellatus (Carv., 1945) Reuteroscopus Kirkaldy, 1905

R. carmelitanus Carv., 1984

* R. cisandinus Carv., 1984

Rhinacloa Reuter, 1876

* R. clavicornis (Reuter, 1905)

* R. forticornis Reuter, 1876

* R. maiuscula Carv., 1948

R. pallidipes Maldonado, 1969 Spanogonicus argentinus (Berg, 1883)

Tapuruyunus acangatus Carv., 1946

* Tytthus neotropicalis (Carv., 1954)

Pilophorini

Sthenaridea Reuter, 1885 Croton sp.; Quercus sp. Solanaceae

(1)

(n)

,

PLANTA-HOSPEDEIRA

Eupatorium sp.; Corylus sp.;

$\underline{\text { Ulmaceae; Euphorbiaceae }}$
Ipomoea sp.; Crotalaria sp.; Daphne sp.; Eleusine sp.; Setaria sp.; Sorghum vulgare

Urera sp.

Solanum cernuum

Croton floribundus; Croton sp.;

Solanum verbascifolium; Solanum sp.

Croton floribundus; Tragia sp. Amaranthus spinosus; Ambrosia sp.; Helianthus annuus; Parthnium sp.; Lantana camara

Enterolobium sp.

Gossypium hirsutum; Solanum melongena

Cajanus cajanus; Medicago sativa; Mimosa sp.; Phaseolus vulgaris; Gossypium sp.

\section{Cajanus cajanus}

Artemisia sp.; Phaseolus vulgaris,

Oryza sp.; Gossypium sp.

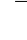

\author{
TRIBO/ESPÉCIE \\ S. carmelitana (Carvalho, 1948) \\ Resthenini \\ Callichilella mesoscutellata \\ (Carv., 1949) \\ C. nigroscutellatus (Carv., 1949) \\ * Cephaloresthenia alvarengai \\ (Carv., 1988)
}

C. oranensis (Carv. \& Carp.,

1987)

Chiloxionotus minensis Carv. \&

Fontes, 1971

Kamaiurana parauara Carv.,

1980

Prepops Reuter, 1905

P. adluteips Carv., 1988

P. caracensis Carv., 1975

P. concinnoides Carv., 1988

* P. concinnus (Stäl, 1860)

P. cruciferus (Berg, 1878)

* P. flavoniger (Stäl, 1860)

P. fragosoi Carv., 1988

P. minensis Carv. \& Fontes, 1970

P. piraporanus Carv., 1988

P. riodocensis Carv., 1988

* P. subsimilis (Reuter, 1907)

* P. tupianus Carv. \&Fontes, 1970

P. vissosensis Carv., 1988

* P. zetterstedti (Stäl, 1860)

Pygophorisca bituberculata

Carv. \& Wall., 1978

Stenodemini

Collaria husseyi Carv., 1955

* C. oleosa (Distant, 1883)

* Collaria scenica (Stäl, 1859)

Dolichomiris Reuter, 1882

* D. linearis Reuter, 1882

* Neotropicomiris costalis Carv. \&

Fontes, 1969

Neotropicomiris longirostris

Carv. \& Fon., 1969

* Opisthocoris carmelitanus Carv. \& Costa, 1991

Trigonotylus Fieber, 1858

T. tenuis Reuter, 1893

Surinamellini

Eustictus Reuter, 1909

Eustictus goianus Carv., 1952

Termatophylini

Termatophylidea Reuter \& Predadores; Asteraceae

Poppius, 1912

* T. opaca Carvalho, 1955

Rhynchospora sp.; Scirphus sp.;

Sclerlia sp.; Cajanus cajan;

Cynodon dactylon; Juncus sp.

Phaseolus vulgaris

Gramineae
PLANTA-HOSPEDEIRA

Cyperus rotundus; Sorghum vulgare; Zea mays; Panicum maximum; $P$. numidianum

Quercus sp.; Salix sp.

Ludwigia octovalvis; L. peruviana

$\underline{\text { Gramineae; }}$ Digitaria sp.; Panicum sp.; Phaseolus vulgaris; $\underline{\text { Setaria }}$ sp.; Sorghum vulgare; Triticum sp.; Zea mays

Avena sativa; Oryza sativa; Panicum numidianum; Pennisetum clandestinum; Stenotaphrum secundatum; Triticum sp.; Zea mays

Cymbopogon sp; Panicum maximum

Gramineae; Aristidea sp.; Chloris $\mathrm{sp}$.

Achnatherum splendens; Agropyron sp.; Cynodon dactylon; Eleusine indica; Zea sp.; Juncus sp.; Brassicaceae Gramineae; Chloris sp.

$\underline{\text { Carya illinoensis; }}$ Populus sp.; $\underline{\text { Salix }}$ sp.

Predador; Eriobotrya japonica; Sarcocephalus exculetus 
Tabela II. Relação das espécies de plantas potencialmente hospedeiras de Miridae no Estado de Minas Gerais (nomes vulgares, famílias e ordens). Espécie (nome vulgar) Família (ordem)

Acacia sp.

Fabaceae (Fabales) Espécie (nome vulgar) Família (ordem)

Acalypha brasiliensis W. Muell. Euphorbiaceae (Euphorbiales)

Achillea sp.

Asteraceae (Asterales)

Achnatherum splendens (Trin.) Gramineae (Poales)

Nevski

Aclandiae sp.

Orchidaceae (Orchidales)

Amaranthus spinosus L.(Caruru)

Amaranthus sp.

Ambrosia sp.

Ananas comosus Merr.

Anemone sp.

Antennaria sp.

Arenaria sp.

Aristida sp.

Artemisia sp.

Atriplex sp.

Avena sativa L. (Aveia)

Baccharis sp.

Balanites aegyptica Wall.

Berberis cretica Falk

B. fremontii Torr.

B. trifoliata Hartw. ex Lindl

Beta vulgaris L.(Beterraba)

Bidens pilosa L. (Picão)

Billbergia sp.

Boerhavia sp.

Borreria sp.

Brassica napus L. (Nabo)

Brassica sp. (Mostarda)

Buxus sempervirens L.

Amaranthaceae (Caryophyllales)

Amaranthaceae (Caryophyllales)

Asteraceae (Asterales)

Bromeliaceae (Bromeliales)

Ranunculaceae (Ranunculales)

Asteraceae (Asterales)

Caryophyllaceae (Caryophyllales)

Gramineae (Poales)

Asteraceae (Asterales)

Echinops sp.

Asteraceae (Asterales)

Eleusine indica Gaertn. (Capim pé- Gramineae (Poales )

de-galinha)

Enterolobium sp. $\quad$ Fabaceae (Fabales)

Ephedra sp.

Epidendrum sp.

Ephedraceae (Ephedrales)

Erigeron sp.

Orchidaceae (Orchidales)

Eriobotra Asteraceae (Asterales)

Erythrina sp. $\quad$ Fabaceae (Fabales)

Eugenia sp. Myrtaceae (Myrtales)

Eupatorium sp. Asteraceae (Asterales)

Fuchsia regia Van. ex. (Vell.) Munz Oenotheraceae (Myrtiflorae)

Glycine hispida Maxim. (Soja) Fabaceae (Fabales)

Gomeza sp. $\quad$ Orchidaceae (Orchidales)

Chenopodiaceae (Caryophyllales) Gossypium hirsutum Cav.(Algodoeiro) Malvaceae (Malvales)

Gramineae (Poales) Haplopappus sp. Asteraceae (Asterales)

Asteraceae (Asterales)

Zygophyllaceae (Geraniales)

Berberidaceae (Ranunculales)

Berberidaceae (Ranunculales)

Berberidaceae (Ranunculales)

Chenopodiaceae (Caryophyllales)

Asteraceae (Asterales)

Bromeliaceae (Bromeliales)

Nyctaginaceae (Caryophyllales)

Rubiaceae (Gentianales)

Cruciferae (Capparales)

Cruciferae (Capparales)

Buxaceae (Celastrales)

Cajanus cajanus Druce (FeijãoGuandu) Fabaceae (Fabales)

Caladium spp. (Tinhorão) Araceae (Arales)

Callitris sp. Cupressaceae (Pinales)

Canavalia sp.

Fabaceae (Fabales)

Helianthus annuus L. (Girassol) Asteraceae (Asterales)

Heliconia psittacorum Sessé \& Musaceae (Zingiberales)

Moç. (Banana-do-mato)

Heliotropium curassavicum L. Boraginaceae (Tubiflorae)

Hibiscus esculentus L. (Quiabo) Malvaceae (Malvales)

Hypericum sp. Guttiferae (Guttiferales)

Indigofera tinctoria Chapm. Fabaceae (Fabales)

Inga sp.

Ipomoea batatas Poir. (Batata) Convolvulaceae (Tubiflorae)

I. corymbosa Roth

Juglans regia L. (Nogueira)

Juncus sp.

Juniperus sp.

Laelia perrinii Bateman

Lantana camara L. (Carambá)

Leonotis nepetaefolia Schimp.

ex Benth.(Cordão-de-frade)

Limonium sp.

Ludwigia octovalvis (Jarq.) Raven

L. peruviana (L.) Hara

Fabaceae (Fabales)

Convolvulaceae (Tubiflorae)

Juglandaceae (Juglandales)

Juncaceae (Juncales)

Cupressaceae (Pinales)

Orchidaceae (Orchidales)

Verbenaceae (Lamiales)

Labiatae (Lamiales)

Pecan)

Cassia senna L. (Sene do Campo) Fabaceae (Fabales)

Cattleya labiata Lindl.

Orchidaceae (Orchidales)

Caucanthus auriculatus Nied. Malpighiaceae (Polyganales)

Lycaste sp.

Plumbaginaceae (Plumbaginales)

Orchidaceae (Orchidales)

Lycopersicum esculentum Mill. Solanaceae (Scrophulariales)

(Tomateiro)

$\begin{array}{lll}\text { Moraceae (Urticales) } & \text { Malus sylvestris Mill. (Macieira) } & \text { Rosaceae (Rosales) }\end{array}$

$\begin{array}{ll}\text { Cecropia sp. (Embaúba) } & \text { Moraceae (Urticales) }\end{array}$

Chenopodium sp.

Chenopodiaceae (Caryophyllales)

Gramineae (Poales)

Cistaceae (Violales)

Cistus villosus $\mathrm{L}$.

Clematis sp.

Ranunculaceae (Ranunculales)

Capparidaceae (Papaverales)

Clerodendrum sp.

Verbenaceae (Lamiales)

Coffea arabica Benth. (Cafeeiro) Rubiaceae (Gentianales)

Colocasia esculenta Schott (Inhame) Araceae (Arales)

Combretum sp.

Commelina diffusa Burm.f.

Corylus sp.

Combretaceae (Myrtiflorae)

Commelinaceae (Commelinales)

Cucurbitaceae (Cucurbitales)

Crotonfloribundus Spreng.(Capixingui) Cucurbitaceae (Cucurbitales)

Cucumis melo reticulata Blanco Cucurbitaceae (Cucurbitales)

Cucurbita maxima Wall.

Cucurbitaceae (Cucurbitales)

Cucurbita moschata Duchesne ex Cucurbitaceae (Cucurbitales)

Poir.

C. pepo Lour. (Aboboreira)

Cucurbitaceae (Cucurbitales)

Cupressus sp.

Cymbopogon sp.

Cupressaceae (Pinales)

Graminaceae (Poales)

Cynodon dactylon Pers.(Capim-de- Graminaceae (Poales)

burro)

Cyperus rotundus Hook.f.

Daphne sp.

Daucus carota L. (Cenoura)

Cyperaceae (Cyperales)

Malus sp.

Rosaceae (Rosales)

Malvastrum coromandelianum Malvaceae (Malvales)

Regel

Manihot utillissima Pohle (Mandioca) Euphorbiaceae (Euphorbiales)

Medicago sativa L. (Alfafa) Fabaceae (Fabales)

Mimosa sp.

Nicotiana tabacum L.(Fumo) Solanaceae (Scrophulariales)

Olea europaea L. (Oliveira) Oleaceae (Oleales)

Oncidium sp.

Oryza sativa L. (Arroz)

Osmanthus sp.

Orchidaceae (Orchidales)

Gramineae (Poales)

Panicum maximum Hochst. ex A. Gramineae (Poales)

Rich. (Capim Colonião)

$P$. numidianum Lam. (Capim Gramineae (Poales)

d'angola)

Parthenium sp. Asteraceae (Asterales)

Pennisetum clandestinum Hochst. ex Gramineae (Poales)

Chiov.

Persea gratissima Gaertn.f. (Abacate) Lauraceae (Magnoliales)

Petroselinum sativum Hoffman Umbelliferae (Araliales)

(Salsa)

Petunia sp.

Solanaceae (Scrophulariales)

Thymelaeraceae(Thymelaeales) Phaseolus vulgaris Wall. (Feijão) Fabaceae (Fabales)

Umbeliferae (Araliales) Philodendron sp.

Phlox sp.

Phoradendron sp.

Pinus sp.

Deudrobium sp.

Asteraceae (Asterales)

Orchidaceae (Orchidales)

Digitaria sp.

Gramineae (Poales)

Piper sp.

Araceae (Arales)

Polemoniaceae (Polemolinales)

Loranthaceae (Santalales)

Pinaceae (Pinales)

Piperaceae (Piperales) 
Tab. II. Continuação

\begin{tabular}{|c|c|}
\hline Espécie (nome vulgar) & Família (ordem) \\
\hline Platanus sp. & Platanaceae (Rosales) \\
\hline Podocarpus sp. & Podocarpaceae (Pinales) \\
\hline Populus nigra L. (Álamo-preto) & Salicaceae (Salicales) \\
\hline P. tremula L. (Choupo tremedor) & Salicaceae (Salicales) \\
\hline Primavera sp. & Orchidaceae (Orchidales) \\
\hline Prosopis juliflora DC. & Fabaceae (Fabales) \\
\hline $\begin{array}{l}\text { Pteridium aquilinum (L.) Kuhn } \\
\text { (Samambaia-das-taperas) }\end{array}$ & Dennstaedtiaceae (Filicales) \\
\hline Pyrus communis L. (Pereira) & Rosaceae (Rosales) \\
\hline Quercus suber Kotschy & Cupuliferae (Fagales) \\
\hline Rhynchospora sp. & Cyperaceae (Cyperales) \\
\hline Ricinus communis $\mathrm{L}$ & Euphorbiaceae (Euphorbiales) \\
\hline Rosmarinus officinalis L. (Alecrim) & Labiatae (Lamiales) \\
\hline Rubus fructicosus Pollich & Rosaceae (Rosales) \\
\hline Rumex sp. (Polygonales) & Polygonaceae \\
\hline Salicornia sp. & Chenopodiaceae (Caryophyllales) \\
\hline Salix sp. & Salicaceae (Salicales) \\
\hline Salvia sp. & Labiatae (Lamiales) \\
\hline Santolina chamaecyparissus L. & Asteraceae (Asterales) \\
\hline $\begin{array}{l}\text { Sarcocephalus exculetus Afeel. ex } \\
\text { Sabine }\end{array}$ & Rubiaceae (Gentianales) \\
\hline Scirphus sp. & Cyperaceae (Cyperales) \\
\hline Scleria sp. & Cyperaceae (Cyperales) \\
\hline Sechium edule Sw. (Chuchuzeiro) & Cucurbitaceae (Cucurbitales) \\
\hline Setaria sp. & Gramineae (Poales) \\
\hline $\begin{array}{l}\text { Sida cordifolia Forssk. (Guaxuma } \\
\text { Branca) }\end{array}$ & Malvaceae (Malvales) \\
\hline $\begin{array}{l}\text { Solanum cernuum Vell. (Braço-de- } \\
\text { preguiça) }\end{array}$ & Solanaceae (Scrophulariales) \\
\hline S. melongena Wall. (Beringela) & Solanaceae (Scrophulariales) \\
\hline Spartium sp. & Fabaceae (Fabales) \\
\hline $\begin{array}{l}\text { Stenotaphrum secundatum (Van der } \\
\text { Walt) Kuntze (Grama Inglesa) }\end{array}$ & Gramineae (Poales) \\
\hline Symphoricarpos sp. & Caprifoliaceae (Dipsacales) \\
\hline Tamarindus indica L.(Tamarindo) & Fabaceae (Fabales) \\
\hline Tamarix sp. & Tamaricaceae (Violales) \\
\hline Tanacetum vulgare $\mathrm{L}$. & Asteraceae (Asterales) \\
\hline Taxodium distichum A.G. Richt. & Taxodiaceae (Pinales) \\
\hline Tecoma stans Juss. & Bignoniaceae (Scrophulariales) \\
\hline Teobroma cacao L. (Cacaueiro) & Rubiaceae (Gentianales) \\
\hline Thevetia sp. (Espirradeira) & Apocynaceae (Gentinales) \\
\hline $\begin{array}{l}\text { Thymus vulgaris Sibth. \& Sm. } \\
\text { (Tomilho) }\end{array}$ & Labiatae (Lamiales) \\
\hline Tillandsia usneoides L. & Bromeliaceae (Bromeliales) \\
\hline Tradescantia fluminensis Vell. & Commelinaceae (Commelinales) \\
\hline Tragia sp. & Euphorbiaceae (Euphorbiales) \\
\hline Triticum sp. (Trigo) & Gramineae (Poales) \\
\hline $\begin{array}{l}\text { Triumfetta semitriloba Jacq. } \\
\text { (Vassourinha) }\end{array}$ & Tiliaceae (Malvales) \\
\hline Urera sp. & Urticaceae (Urticales) \\
\hline Urtica sp. & Urticaceae (Urticales) \\
\hline Verbascum sp. & Scrophulariaceae (Scrophulariales) \\
\hline Verbena sp. & Verbenaceae (Lamiales) \\
\hline Vicia sp. & Fabaceae (Fabales) \\
\hline $\begin{array}{l}\text { Vigna sinensis Endl. ex Hassk. } \\
\text { (Feijão chicote) }\end{array}$ & Fabaceae (Fabales) \\
\hline Zea mays L. (Milho) & Gramineae (Poales) \\
\hline
\end{tabular}

As famílias de plantas hospedeiras listadas apresentam importância econômica destacada. Fabaceae (ordem Fabales) formada por espécies de grande valor econômico como alimento (amendoim, grão-de-bico, lentilha, ervilha, soja, feijão) ou forrageiras (alfafa), além de um grande número de invasoras de culturas e pastagens. Asteraceae (Asterales) inclui as "compostas", com espécies de uso medicinal (camomila, carqueja, erva-grossa, picão, alecrim-do-campo, arnica), alimentar (girassol, alcachofra, almeirão, alface) e ornamental. Solanaceae (Scrophulariales) uso na alimentação (pimenta, pimentão, giló, beringela, batata-inglesa, tomate), medicinal (beladona, meimendro, ervamoura, jurubeba, estramônio), ornamental (dama-da-noite) e industrial (fumo). Malvaceae (Malvales) de uso ornamental (lanterna-japonesa, hibisco), industrial (algodoeiro), medicinal (malva) e alimentar (quiabo). Gramineae (Poales) inclui espécies de uso na alimentação (trigo, cevada, centeio, arroz, cana-de-açúcar, milho, aveia), indústria (piaçaba, sapé), forrageira (capim, sorgo), medicinal (erva-cidreira) e ornamental (grama, bambu). Convolvulaceae (Tubiflorae) inclui espécies de uso alimentar (batata-doce) e ornamental (ipoméia, flor-decardeal). Euphorbiaceae (Euphorbiales) de uso medicinal (quebra-pedra, cróton), ornamental (nogueira-brasileira, acalifa, florde-papagaio, coroa-de-Cristo), industrial (seringueira) e alimentar (mamona, mandioca). Orchidaceae (Orchidales) é fornecedora de exemplares ornamentais (orquídeas) e de uso na alimentação (baunilha). As outras ordens também têm considerável aproveitamento econômico. Gentianales têm representantes ornamentais, como a alamanda (Apocynaceae), medicinais, como o genipapo (Rubiaceae), industrial, peroba (Apocynaceae), além, é claro, do café (Rubiaceae), cultura das mais destacadas do país. Caryophyllales têm representantes ornamentais, como o craveiro (Caryophyllaceae) e a crista-de-galo (Amaranthaceae), medicinais, erva-de-Santa-Maria (Chenopodiaceae), além do espinafre e da beterraba (Chenopodiaceae), de uso alimentar.

Em conclusão, o acréscimo significativo do número de espécies para Minas Gerais 


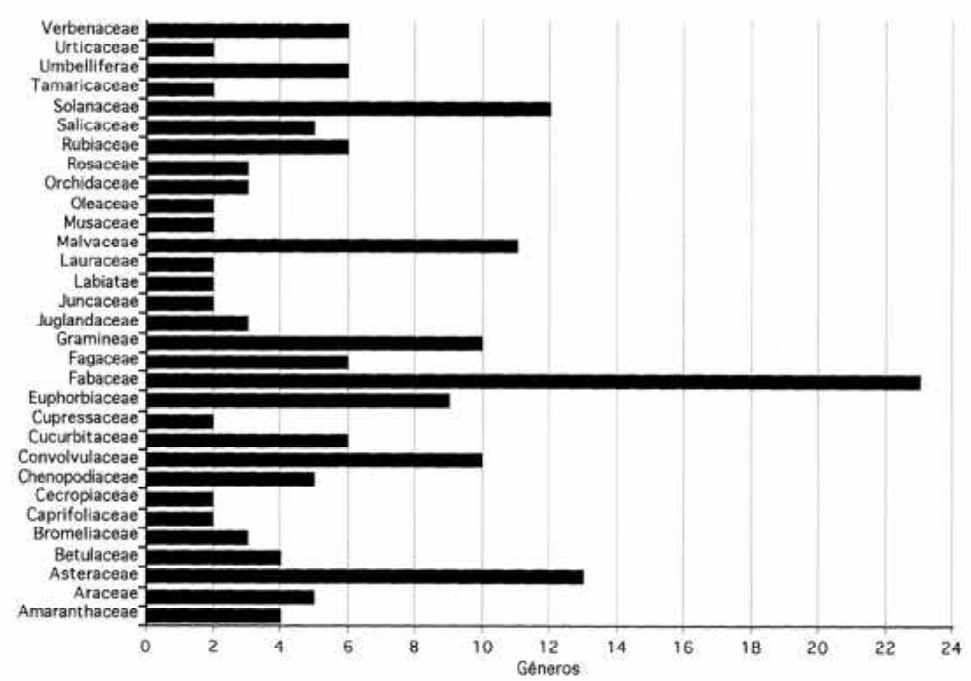

Fig. 3. Famílias de plantas hospedeiras de Miridae, Minas Gerais e o número de gêneros de mirídeos. Somente foram incluídas famílias com dois ou mais gêneros.

veio dar ao Estado o status de maior biodiversidade de Miridae para o país. Esta realidade pode ser modificada quando forem realizados maiores estudos faunísticos em outras localidades brasileiras. Ainda é muito pouco o registro do número de plantas-hospedeiras se levarmos em consideração o número de espécies de mirídeos conhecido. No entanto, os novos registros sobre plantas hospedeiras sugerem mudanças de hábitos comportamentais das espécies, principalmente fitosuccívoras, possivelmente resultado da ação antrópica no meio agrícola. A tentativa de se estabelecer hipóteses de "plantas potencialmente hospedeiras", principalmente para gêneros de mirídeos (tab.I), vem possibilitar a previsão de possíveis novas plantas hospedeiras de importância econômica.

Agradecimentos. À Fundação de Amparo à Pesquisa do Estado de Minas Gerais (FAPEMIG) pelo suporte financeiro no projeto CBS 288/97; à Marina do Carmo Santana de Carvalho, Secretaria de Agricultura, Prefeitura Municipal de Campos Altos, MG; ao Heron Reger de Carvalho, Presidente da Cooperativa de Campos Altos, MG; ao Departamento Técnico da COOPADAP, São Gotardo, MG; ao José Elias Said de Resende, Professor e Diretor de Extensão da CEDAF, Florestal, MG; à Cooperativa Agropecuária de Dores do Indaiá, MG, e a todas aquelas cooperativas e prefeituras mineiras visitadas que nos deram apoio.

\section{REFERÊNCIAS BIBLIOGRÁFICAS}

Brummitt, R. K. \& Powell, C. E. eds. 1996. Authors of plant names. Kew, Royal Botanic Gardens. 732 p.

Ferreira, P. S. F. \& Martins, D. S. 1982. Contribuição ao método de captura de insetos por meio de armadilha luminosa, para obtenção de exemplares sem danos morfológicos. Revta Ceres, Viçosa, 29 (165):538-543.

Gemtchúsnicov, I. D. 1976. Manual de taxonomia vegetal: plantas de interesse econômico. São Paulo, Agronômica Ceres. 368 p.

Henry, T. J. 2000. The predatory Miridae: a glimpse at the other plant bugs. In: ShePherd, M. Wings (essays on invertebrates conservation). Portland, Xerces Society. p. 17-20.

Henry, T. J. \& Froeschner, R. C. eds. 1988. Catalog of the Heteroptera or true bugs, of Canada and the

Continental United States. New York, E. J. Brill. 958 p. 
Sснин, R. T. 1995. Plant bugs of the world (Insecta: Heteroptera: Miridae). Systematic catalog, distributions, host list, and bibliography. New York, The New York Entomological Society. 1329 p.

Schultz, A. 1990. Introdução à botânica sistemática. 6 ed. Porto Alegre, Universidade Federal do Rio Grande do Sul. 414 p.

Wheeler, A. G., JR. 2000a. Plant bugs (Miridae) as plant pest. In: Schaefer, C. W. \& Panizzi, A. R. Heteroptera of Economic Importance. Boca Raton, CRS. p. 37-83.

.2000b. Predacious plant bugs (Miridae). In: Schaefer, C. W. \& PAnIzzi, A. R. Heteroptera of Economic Importance. Boca Raton, CRS. p. 657-693.

Recebido em 17.02.2000, aceito em 18.05.2001. 